\title{
Qualidade de painéis LVL produzidos com madeira de clones de Eucalyptus urophylla
}

José Benedito Guimarães Junior ${ }^{1}$, Thiago de Paula Protásio ${ }^{1 *}$, Rafael Farinassi Mendes ${ }^{2}$, Lourival Marin Mendes ${ }^{3}$, Bárbara Maria Ribeiro Guimarães ${ }^{3}$, Humberto Fauller de Siqueira ${ }^{1}$

${ }^{1}$ Universidade Federal de Goiás, Regional Jataí, Câmpus Jatobá, C.P. 3, Rodovia BR-364, km 192, Parque Industrial, CEP 75801-615, Jataí, GO, Brasil 2Universidade Federal de Lavras, Departamento de Engenharia, C.P. 3037, CEP 37200-000, Lavras, MG, Brasil

${ }^{3}$ Universidade Federal de Lavras, Departamento de Ciências Florestais, C.P. 3037, CEP 37200-000, Lavras, MG, Brasil

"Autor correspondente:

depaulaprotasio@gmail.com

Termos para indexação:

Propriedades físicas e mecânicas

Painéis laminados

Resorcinol-formaldeído

Index terms:

Physical and mechanical properties

Laminated panels

Resorcinol-formaldehyde

Histórico do artigo:

Recebido em 09 out 2014

Aprovado em 03 set 2015

Publicado em 30 set 2015

doi: $10.4336 / 2015 . p f b .35 .83 .797$
Resumo - O objetivo deste trabalho foi verificar a qualidade de painéis LVL produzidos com a madeira de cinco clones de Eucalyptus urophylla. Para cada clone foram produzidos cinco painéis LVL, com lâminas de $2 \mathrm{~mm}$ de espessura, adesivo resorcinolformaldeído com gramatura de $320 \mathrm{~g} \mathrm{~m}^{-2}$, pressão de 1,47 MPa durante o período de 8 $\mathrm{h}$ a temperatura ambiente. De maneira geral, todos os clones apresentaram potencial para a produção de painéis LVL, com destaque para o clone 26, por apresentar melhor desempenho com relação às propriedades físicas e resistência à colagem.

\section{Quality of LVL panels produced with wood from Eucalyptus urophylla clones}

\begin{abstract}
The objective of this research was to verify the quality of LVL panels manufactured with wood of five clones of Eucalyptus urophylla. Five LVL panels were produced for each clone, with $2 \mathrm{~mm}$ veneer, resorcinol-formaldehyde adhesive with a $320 \mathrm{~g} \mathrm{~m}^{-2}$ weight, $1.47 \mathrm{MPa}$ pressure for a period of $8 \mathrm{~h}$ at room temperature. In general, all clones showed potential to be used for production of LVL panels, especially clone 26 , as it showed better performance regarding physical properties and bonding strength.
\end{abstract}

\section{Introdução}

Os compostos estruturais de madeira compreendem inúmeros produtos, como os painéis LVL, os quais são utilizados predominantemente em países onde há tradição no uso de madeira em sistemas construtivos (Müller et al., 2015). No Brasil, a produção desses painéis ainda é incipiente e a sua aplicação como elemento estrutural não foi ainda viabilizada (Iwakiri et al., 2008).
Nesse sentido, pesquisas estão sendo realizadas visando o uso de espécies florestais plantadas de rápido crescimento, consideradas alternativas ao uso de espécies nativas, de modo a viabilizar a aplicação desses painéis na construção civil (Pedrosa et al., 2005; Iwakiri et al., 2008, 2010; Bortoletto Júnior, 2009; Palma \& Ballarin, 2011; Pio et al., 2012; Müller et al., 2015).

Segundo Pedrosa et al. (2005), as densidades médias a baixas das madeiras das espécies florestais plantadas atualmente no Brasil e, utilizadas precocemente na produção de compósitos, deixam de ser restrições 
para uso estrutural, por meio do emprego de adesivos específicos e demais variáveis inerentes ao processo de manufatura dos painéis.

Aliado a isso, a crescente necessidade de se utilizar elementos estruturais de qualidade controlada e mais homogêneos, juntamente com o marcante avanço na tecnologia dos adesivos e a disponibilidade de madeira de florestas plantadas, contribuíram para o surgimento de novos materiais de construção, dentre estes, destacam-se os produtos engenheirados de madeira, como flanges de vigas em perfil "I" que podem ser produzidas a partir de painéis LVL (Pedrosa et al., 2005; Iwakiri et al., 2010; Pio et al., 2012).

Segundo a norma Standard Specification for Evaluation of Structural Composite Lumber Products (American Society for Testing and Material, 2001), o LVL (laminated veneer lumber) pode ser definido como um composto estrutural constituído de lâminas de madeira de pequena espessura, com as fibras da madeira orientadas ao longo do comprimento da peça, no qual a espessura das lâminas não deve exceder $6,4 \mathrm{~mm}$.

O painel laminado unidirecional foi desenvolvido nos EUA na década de 1970, com denominação comercial de LVL. A sua aplicação destina-se principalmente ao uso estrutural, como: pisos de carrocerias, vagões de trens, escadas, vigas, flanges de vigas em "I", entre outras, em função da maior resistência na direção longitudinal ao plano do painel (Iwakiri, 2005).

De acordo com Pease (1994), destacado também por Iwakiri et al. (2008), os painéis LVL apresentam as seguintes vantagens em comparação à madeira sólida: (a) maior resistência em decorrência da classificação das lâminas e do controle das variáveis do processo de manufatura; (b) flexibilidade dimensional - os painéis podem ser fabricados com qualquer largura e comprimento desejado; (iii) utilização de inúmeras espécies florestais.

O LVL distingue-se do compensado pelo fato das lâminas serem coladas, umas sobre as outras, com as fibras dispostas paralelamente. A produção comercial do LVL tem empregado lâminas com 3,2 mm de espessura, resina à base de fenol-formaldeído e prensagem a quente convencional. O LVL pode também ser produzido através da prensagem contínua, em larguras de 100 a $1.200 \mathrm{~mm}$, espessuras de 19 a $75 \mathrm{~mm}$ e comprimentos de até $25 \mathrm{~m}$ (Walker, 1993).
Palma \& Ballarin (2011) destacaram que o LVL é fabricado principalmente a partir de madeiras de coníferas, com predominância de Pinus. Hoje, com a diminuição em nível mundial da oferta de madeira de grandes diâmetros vinda de florestas nativas, seja por razões ecológicas ou esgotamento devido à exploração indiscriminada, as indústrias de laminados têm optado por espécies oriundas de reflorestamentos, como Pinus, Eucalyptus, Populus, dentre outras.

Uma das alternativas na busca de se produzir matériaprima com qualidade adequada para a produção de diferentes produtos a base de madeira, principalmente os laminados, é trabalhar as variações existentes entre espécies, procedências, famílias e clones. Essa pode ser uma alternativa para se obter um produto de melhor qualidade a partir da madeira de eucalipto, uma vez que a maioria de suas propriedades, consideradas limitantes na produção de painéis reconstituídos, encontram-se sob moderado ou alto controle genético. Iwakiri et al. (2006) reforçam a ideia de Tsoumis (1991), relatando que as características tecnológicas da madeira adequada para laminação estão relacionadas, principalmente, com a densidade da madeira (baixa a média), as características do fuste (diâmetro e forma) e a grã (direita a levemente inclinada).

Almeida et al. (2004) afirmaram que as buscas por novas fontes de matérias-primas somadas às vantagens das madeiras de Eucalyptus, demonstram a necessidade de aumentar o volume de pesquisas sobre as espécies, híbridos e clones deste gênero e também sobre a qualidade dos produtos oriundos destes.

Neste sentido, o objetivo deste trabalho foi verificar a qualidade de painéis LVL produzidos com madeira de diferentes clones de Eucalyptus urophylla S. T. Blake.

\section{Material e métodos}

Foram utilizadas árvores de cinco clones de Eucalyptus urophylla S. T. Blake com 94 meses de idade, plantadas pela Companhia Mineira de Metais - Unidade Agroflorestal (VM-AGRO) no município de Vazante, região noroeste de Minas Gerais. Para cada clone foram amostradas cinco árvores.

A área está situada a $17^{\circ} 36^{\prime} 09^{\prime \prime}$ Sul e $46^{\circ} 42^{\prime} 42^{\prime \prime}$ Oeste em altitude de $550 \mathrm{~m}$. Segundo a classificação de Köppen, o clima é do tipo Aw, tropical úmido de savana, 
com inverno seco e verão chuvoso. A temperatura média anual é de $24^{\circ} \mathrm{C}$ e a precipitação média anual acumulada é de $1.450 \mathrm{~mm}$.

Na Tabela 1 são apresentadas as características médias dos clones, tais como diâmetro a 1,30 m do solo (DAP) e densidade básica.

As lâminas de madeira foram obtidas em torno laminador, após o aquecimento das toras a temperatura de $66{ }^{\circ} \mathrm{C}$, por um período de $24 \mathrm{~h}$ em água, de acordo com recomendações de Iwakiri (2005). Elas foram geradas com espessura nominal de $2 \mathrm{~mm}$ e guilhotinadas nas dimensões de $500 \mathrm{~mm}$ x $500 \mathrm{~mm}$ (largura $\mathrm{x}$ comprimento).

Tabela 1. Diâmetro a 1,30 m do solo e densidade básica de clones de Eucalyptus urophylla.

\begin{tabular}{ccc}
\hline Clone & DAP (cm) & $\begin{array}{c}\text { Densidade básica média } \\
\left(\mathbf{g ~ c m}^{-3}\right)\end{array}$ \\
\hline 19 & 34,69 & 0,650 \\
26 & 34,37 & 0,600 \\
28 & 32,15 & 0,520 \\
36 & 37,24 & 0,590 \\
58 & 32,91 & 0,560 \\
\hline
\end{tabular}

As lâminas foram tabicadas no plano horizontal para secagem natural, em local coberto, até atingirem a umidade de equilíbrio higroscópico (umidade entre 10\% e $12 \%$ ). Posteriormente, tiveram este valor reduzido para $8 \%$, por meio de secagem em estufa com circulação forçada de ar. Anteriormente à manufatura dos painéis LVL, as lâminas secas foram classificadas em categorias de qualidade decrescente (A, B, C e D), conforme a Norma Brasileira NBR 9531 (Associação Brasileira de Normas Técnicas, 1986). No presente estudo, foram utilizadas as lâminas com a classificação B para as capas, e lâminas com classificação $\mathrm{C}$ para o miolo dos painéis.

O painel foi composto por nove lâminas com dimensões de 2,0 mm x $480 \mathrm{~mm}$ x $480 \mathrm{~mm}$ (espessura x largura $\mathrm{x}$ comprimento). Para cada clone avaliado foram confeccionados cinco painéis LVL. O adesivo utilizado na produção dos painéis foi o resorcinol-formaldeído, com gramatura de $320 \mathrm{~g} \mathrm{~m}^{-2}$. Os painéis foram prensados com uma pressão de $1,47 \mathrm{MPa}$ durante o período de $8 \mathrm{~h}$ a temperatura ambiente.

$\mathrm{Na}$ avaliação das propriedades físicas, foram retirados quatro corpos de prova por painel para densidade aparente, quatro para umidade e quatro para absorção de água, determinados de acordo com a norma ASTM
D 1037 (American Society for Testing and Material, 1999). Para os ensaios mecânicos, foram retirados quatro corpos de prova por painel para ensaios de flexão estática e doze corpos de prova por painel para ensaios de resistência da linha de cola nos teste seco, úmido e pós-fervura. Os ensaios foram realizados de acordo com a Norma Europeia EN 314-2 (European Standard, 1993) para resistência da linha de cola e EN 310 (European Standard, 2002) para flexão estática.

A análise estatística constituiu-se de delineamento inteiramente casualizado, com cinco repetições, sendo cada clone considerado como um tratamento. A análise dos dados se deu mediante a utilização de análise de variância e teste de comparação múltipla de médias Scott-Knott, ambos a 5\% de significância.

\section{Resultados e discussão}

Os valores médios de umidade, absorção de água e densidade aparente dos painéis LVL produzidos com a madeira de clones de Eucalyptus urophylla se encontram na Tabela 2. Observa-se que não houve efeito estatístico significativo de clone para a umidade dos painéis, sendo a média geral de $12,3 \%$.

Tabela 2. Propriedades físicas dos painéis LVL de clones de Eucalyptus urophylla.

\begin{tabular}{cccc}
\hline Clone & $\begin{array}{c}\text { Umidade } \\
(\mathbf{\%})\end{array}$ & $\begin{array}{c}\text { Densidade aparente } \\
\left(\mathbf{g ~ c m}^{-3}\right)\end{array}$ & $\begin{array}{c}\text { Absorção de } \\
\text { água total (\%) }\end{array}$ \\
\hline 19 & $11,80 \mathrm{~A}$ & $0,830 \mathrm{~A}$ & $23,93 \mathrm{C}$ \\
26 & $13,61 \mathrm{~A}$ & $0,790 \mathrm{~A}$ & $22,60 \mathrm{C}$ \\
28 & $12,60 \mathrm{~A}$ & $0,610 \mathrm{D}$ & $32,05 \mathrm{~A}$ \\
36 & $11,96 \mathrm{~A}$ & $0,770 \mathrm{~B}$ & $27,22 \mathrm{~B}$ \\
58 & $11,62 \mathrm{~A}$ & $0,720 \mathrm{C}$ & $28,61 \mathrm{~B}$ \\
\hline $\mathrm{CV}(\%)$ & 4,36 & 1,34 & 3,66 \\
\hline
\end{tabular}

Médias seguidas de mesma letra não diferem entre si pelo teste Scott-Knott $(\alpha=0,05)$.

Para densidade aparente dos painéis nota-se que houve diferença estatística significativa entre os diferentes materiais genéticos estudados, em que os clones 19 e 26 se destacaram com os maiores valores médios e o clone 28 apresentou o menor valor médio. Os resultados encontrados para a densidade aparente dos painéis LVL pode ser explicada pela densidade básica dos clones, pois existe uma relação direta entre essas propriedades estudadas (Tabelas 1 e 2 e Figura 1). 


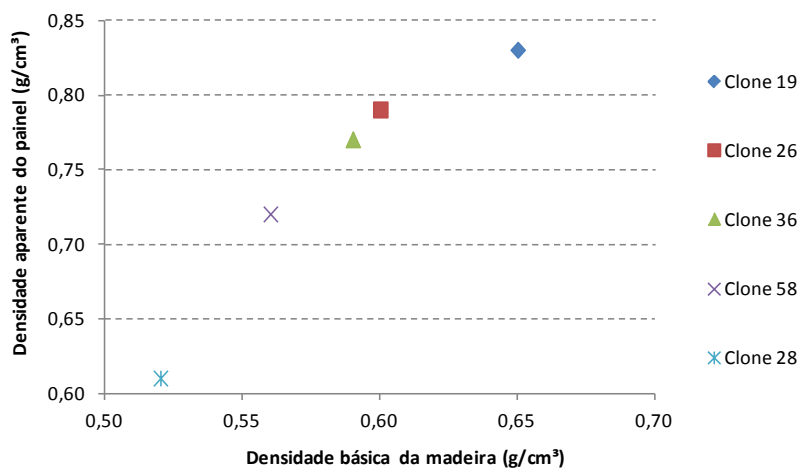

Figura 1. Relação observada entre a densidade básica da madeira e a densidade aparente dos painéis LVL.

Pio et al. (2012), trabalhando com LVL produzidos com madeira de Eucalyptus grandis, nas idades de 15 e 20 anos, observaram densidade aparente dos painéis variando entre 0,635 e $0,784 \mathrm{~g} \mathrm{~cm}^{-3}$; valores estes bem próximos aos observados nesta pesquisa.

Os valores médios de densidade dos LVL foram de 17 a $31 \%$ superiores à densidade da madeira, sendo o menor valor obtido para o clone 28 e o maior valor obtido para o clone 19. Bortoletto Junior (2009), trabalhando com LVL de Pinus merkussi Jungh. e de Vriese, observou aumento de aproximadamente $41 \%$ quando comparou a densidade básica da madeira $\left(0,438 \mathrm{~g} \mathrm{~cm}^{-3}\right)$ com a densidade aparente dos painéis $\operatorname{LVL}\left(0,618 \mathrm{~g} \mathrm{~cm}^{-3}\right)$. Esse autor atribuiu esses resultados à densificação que ocorre durante a prensagem do LVL, bem como ao uso de parte das lâminas classificadas para a manufatura do painel.

Cabe ressaltar que as diferenças entre a densidade dos painéis LVL produzidos com madeira de Eucalyptus, comparativamente às chapas de Pinus relatadas na literatura (Bortoletto Junior, 2009), deve-se aos distintos valores entre a densidade da madeira de origem, sendo que isso pode influenciar consideravelmente as propriedades físicas e mecânicas e, consequentemente, a aplicação do painel como material de construção.

Para a propriedade absorção de água houve diferença estatística significativa entre os diferentes materiais genéticos estudados, sendo observados três grupos homogêneos por meio do teste de Scott-Knott (Tabela 1). Os painéis produzidos com a madeira do clone 28 foram os que absorveram a maior quantidade de água em decorrência da sua baixa densidade aparente, comparativamente aos demais tratamentos avaliados. Suchsland (2004) relata que, assim como para madeira maciça, painéis com menor densidade tendem a absorver mais água por apresentarem menor quantidade de parede celular para um mesmo volume.

Com exceção do painel produzido com madeira do clone 28, os demais apresentaram valores de absorção de água abaixo daqueles encontrados por Melo (2012), trabalhando com madeira de paricá (Schizolobium amazonicum) para produção de LVL e utilizando adesivo poliuretano com gramatura de $200 \mathrm{~g} \mathrm{~m}^{-2}$. O autor observou valor médio para essa propriedade física de $32 \%$.

Esses resultados distintos relatados por Melo (2012) comparativamente aos valores encontrados neste trabalho podem ser explicados analisando-se a densidade dos painéis. $\mathrm{O}$ autor encontrou densidade aparente média para os painéis LVL de $0,428 \mathrm{~g} \mathrm{~cm}^{-3}$. Dessa forma, as chapas produzidas com madeira dos clones 19 e 26 apresentaram densidade aparente superior em, aproximadamente, $89 \%$ comparativamente aos painéis LVL da madeira de paricá.

Adicionalmente, pode-se constatar que a absorção de água total observada para os painéis deste trabalho foram inferiores aos encontrados por Lima et al. (2013) trabalhando com três espécies amazônicas na produção de LVL, com adesivo resorcinol-formaldeído, aplicado com gramatura $160 \mathrm{~g} \mathrm{~m}^{-2}$. Os autores observaram valores variando entre 36,2 a $85,6 \%$. São desejados menores valores para essa propriedade física, portanto, esse resultado reforça o potencial de uso de madeira dos clones de Eucalyptus urophylla avaliados para a produção de painéis LVL.

Na Tabela 3 podem-se verificar os valores médios da resistência ao cisalhamento e falha na madeira, dos diferentes tratamentos, nas condições: seca, úmida e pós-fervura.

Observa-se que não houve diferença estatística significativa entre os clones estudados para o teste de cisalhamento na condição seca. Palma \& Ballarin (2011) encontraram valores médios de tensão ligeiramente superiores aos obtidos nesta pesquisa, variando de 5,96 e 7,00 MPa. É válido mencionar que os autores produziram LVL a partir de madeira de Eucalyptus grandis, adesivo fenol-formaldeído e com gramatura de $350 \mathrm{~g} \mathrm{~m}^{-2}$ (linha dupla). Provavelmente, as diferenças encontradas para o teste de cisalhamento deve-se à gramatura consideravelmente superior à utilizada neste trabalho. 
Lima et al. (2013) observaram valores médios de cisalhamento no teste seco de 5,15; 5,17; 4,04 e 4,71 MPa para painéis LVL produzidos com nove lâminas de freijó (Cordia goeldiana), amapá (Brosimum parinarioides), faveira (Parkia gigantocarpa) e pinus (Pinus oocarpa), gramatura de $160 \mathrm{~g} \mathrm{~m}^{-2} \mathrm{em}$ linha simples e com o mesmo adesivo utilizado neste trabalho (resorcinol-formaldeído). Cabe ressaltar que esses autores não encontraram diferenças estatísticas entre esses painéis e que os valores foram inferiores aos painéis LVL da madeira dos clones de Eucalyptus, provavelmente, em decorrência da gramatura utilizada.

Tabela 3. Valores médios de cisalhamento seco, em água fria e sob fervura dos painéis LVL de clones de Eucalyptus urophylla.

\begin{tabular}{ccccccc}
\hline & \multicolumn{7}{c}{ Cisalhamento } \\
\cline { 2 - 7 } Clone & \multicolumn{2}{c}{ Seco } & \multicolumn{2}{c}{ Úmido } & Pós-Fervura \\
\cline { 2 - 7 } & $\begin{array}{c}\text { Tensão } \\
\text { (MPa) }\end{array}$ & Falha (\%) & Tensão (MPa) & Falha (\%) & Tensão (MPa) & Falha (\%) \\
\hline 19 & $5,3 \mathrm{~A}$ & 67,4 & $4,1 \mathrm{~B}$ & 44,2 & $2,9 \mathrm{~B}$ & 71,6 \\
26 & $6,3 \mathrm{~A}$ & 61,2 & $5,3 \mathrm{~A}$ & 35,8 & $4,2 \mathrm{~A}$ & 64,2 \\
28 & $4,2 \mathrm{~A}$ & 48,6 & $4,7 \mathrm{~B}$ & 45,0 & $3,8 \mathrm{~B}$ & 53,2 \\
36 & $4,9 \mathrm{~A}$ & 79,8 & $4,3 \mathrm{~B}$ & 54,0 & $3,3 \mathrm{~B}$ & 89,0 \\
58 & $6,7 \mathrm{~A}$ & 51,8 & $4,3 \mathrm{~B}$ & 22,4 & $3,6 \mathrm{~B}$ & 81,2 \\
\hline CV (\%) & 23,3 & 9,2 & 12,6 & 25,4 & 12,3 & 9,2 \\
\hline
\end{tabular}

Médias seguidas de mesma letra não diferem entre si pelo teste Scott-Knott, com significância de 5\%.

Foram observadas diferenças significativas para o cisalhamento sob condição úmida e pós-fervura entre os materiais genéticos estudados; com destaque para o clone 26 por apresentar os maiores valores de tensão, com 5,3 e 4,2 MPa nas condições úmida e pós-fervura, respectivamente. Não houve diferença estatística entre os demais clones.

Os valores de cisalhamento na condição úmida ficaram acima dos observados por Lima et al. (2013) trabalhando com LVL de espécie amazônica, madeira de pinus e misturas, obtendo valores variando entre 2,67 e 3,75 $\mathrm{MPa}$. Este resultado pode ser ocasionado principalmente pela gramatura do adesivo utilizado por estes autores $\left(160 \mathrm{~g} \mathrm{~m}^{-2}\right)$ sendo inferior à utilizada neste trabalho $\left(320 \mathrm{~g} \mathrm{~m}^{-2}\right)$.

Para os valores na condição pós-fervura, observa-se que estão próximos daqueles encontrados por Iwakiri et al. (2008), entre 1,61 e 4,5 MPa, ao estudarem painéis LVL produzidos com Eucalyptus grandis Hill ex Maiden e Eucalyptus dunnii Maiden, utilizando adesivo fenolformaldeído, aplicado com gramatura de $360 \mathrm{~g} \mathrm{~m}^{-2} \mathrm{em}$ linha dupla.

De maneira geral, os resultados obtidos no cisalhamento paralelo à linha de colagem, para todos os clones estudados, atenderam aos requisitos mínimos da norma Europeia EN 314-2 (European Standard, 1993), cujo valor é de 1,0 MPa, independente da porcentagem de falha na madeira.
Na Tabela 4 estão apresentados os resultados dos módulos de elasticidade (MOE) e de ruptura (MOR) obtidos na flexão estática, no sentido flatwise, para os painéis LVL produzidos com a madeira dos clones de Eucalyptus urophylla. A posição flatwise é aquela em que as lâminas que compõem os corpos de prova se encontram dispostas em planos horizontais, perpendiculares à direção da força aplicada (Palma \& Ballarin, 2011; Müller et al., 2015). Observa-se que não foram observadas diferenças significativas entre os clones para ambas as propriedades, mesmo sendo observados distintos valores para a densidade aparente dos painéis.

Tabela 4. Valores médios dos módulos de elasticidade (MOE) e de ruptura (MOR) à flexão estática para os clones de Eucalyptus urophylla.

\begin{tabular}{ccc}
\hline Clone & MOE flatwise (MPa) & $\begin{array}{c}\text { MOR flatwise } \\
(\mathbf{M P a})\end{array}$ \\
\hline 19 & $12.271,6 \mathrm{~A}$ & $53,5 \mathrm{~A}$ \\
26 & $11.633,7 \mathrm{~A}$ & $55,3 \mathrm{~A}$ \\
28 & $10.408,6 \mathrm{~A}$ & $54,4 \mathrm{~A}$ \\
36 & $12.558,2 \mathrm{~A}$ & $59,8 \mathrm{~A}$ \\
58 & $11.415,7 \mathrm{~A}$ & $57,2 \mathrm{~A}$ \\
\hline CV $(\%)$ & 9,2 & 6,5 \\
\hline
\end{tabular}

Médias seguidas de mesma letra não diferem entre si pelo teste Scott-Knott, com significância de 5\%. 
Para MOE flatwise observou-se que os valores variaram entre 10.408,6 a 12.558,2 $\mathrm{MPa}$, com média geral de 11.657,56 MPa. Estes ficaram acima dos observados por Pio et al. (2012) de 9.410 MPa para o painel LVL de madeira de Eucalyptus grandis, com densidade aparente de $0,667 \mathrm{~g} \mathrm{~cm}^{-3}$. Guzmán \& Hernandez (2007) encontraram valores de 10.996 MPa para o painel LVL de densidade aparente de $0,575 \mathrm{~g} \mathrm{~cm}^{-3}$ produzido com madeira de Eucalyptus nitens. Provavelmente, os valores superiores de densidade aparente dos painéis produzidos tenham propiciado os melhores valores de MOE na posição flatwise comparativamente às literaturas supracitadas.

No MOR flatwise nota-se que os valores oscilaram entre 53,5 e 59,8 $\mathrm{MPa}$, com média geral de 56,04 $\mathrm{MPa}$. Estes valores foram superiores aos observados por Iwakiri et al. (2010) para painéis LVL de madeira de paricá (Schizolobium amazonicum Huber ex Ducke) de 35,5 MPa e 49,9 MPa. Esses painéis apresentaram densidade aparente de $0,381 \mathrm{e} 0,414 \mathrm{~g} \mathrm{~cm}^{-3}$, respectivamente. Possivelmente, essa baixa densidade aparente dos painéis de paricá reportados na literatura tenha influenciado esses resultados.

Os valores médios de resistência e rigidez mostram que, em geral, os painéis LVL apresentaram um bom desempenho mecânico, quando comparado aquele reportado na norma NBR 7190 (Associação Brasileira de Normas Técnicas, 1997), para a madeira sólida dessa espécie.

\section{Conclusões}

Os painéis LVL produzidos com a madeira dos diferentes clones de Eucalyptus urophylla apresentam potencial tecnológico e comercial.

O clone 26 apresentou comportamento diferenciado, por apresentar melhor desempenho com relação às propriedades físicas e por se destacar dos demais clones no que se refere à resistência à colagem.

\section{Agradecimentos}

Os autores expressam seus sinceros agradecimentos ao $\mathrm{CNPq}$, Fapemig e Capes pelo apoio financeiro, ao Núcleo de Estudos e Pesquisa em Produtos Florestais (NEPPFLOR) da Universidade Federal de Goiás, Regional Jataí, e ao Núcleo de Estudos em Painéis de Madeira (NEPAM) da Universidade Federal de Lavras.

\section{Referências}

ALMEIDA, R. R.; BORTOLETTO JÚNIOR, G.; JANKOWSKY, I. P. Produção de lâminas a partir da madeira de clones do híbrido Eucalyptus grandis x Eucalyptus urophylla. Scientia Forestalis, Piracicaba, n. 65, p. 49-58, 2004.

AMERICAN SOCIETY FOR TESTING AND MATERIALS. ASTM D 1037: standard test methods of evaluating properties of wood-based fiber and particle panel materials. Philadelphia, 1999. $30 \mathrm{p}$.

AMERICAN SOCIETY FOR TESTING AND MATERIALS. ASTM D 5456:standard specification for evaluation of structural composite lumber products. Philadelphia, 2001. 14 p.

ASSOCIAÇÃO BRASILEIRA DE NORMAS TÉCNICAS. NBR 9531: chapas de madeira compensada: classificação. Rio de Janeiro, 1986. 3 p.

ASSOCIAÇÃO BRASILEIRA DE NORMAS TÉCNICAS. NBR 7190: projeto de estruturas de madeira. Rio de Janeiro, 1997. 107 p.

BORTOLETTO JÚNIOR, G. Estudo comparativo das propriedades físicas e mecânicas da madeira e do LVL de Pinus merkusii. Revista Forestal Venezolana, Mérida, v. 53, n. 2, p. 191-195, 2009.

EUROPEAN STANDARD. EN 310: Determination of modulus of elasticity in bending and of bending strength. Lisboa, 2002.

EUROPEAN STANDARD. EN 314-2: plywood: bonding quality: part 2, requirements. Geneva, 1993.

GUZMÁN, F. M.; HERNÁNDEZ, C.G. Propriedades físicas y mecánicas. In: LVL vigas de chapas laminadas confeccionadas com Pinus radiata y Eucalyptus nitens. Concepción: INFOR, 2007. p. 12-32.

IWAKIRI, S.; ALMEIDA, B. C.; BIASI, C. P.; CHIES, D.; GUISANTES, F. P.; FRANZONI, J. A.; RIGATTO, P. A.; BETTEGA, W. P. Avaliação da qualidade do compensado fenólico de Eucalyptus grandis. Ciência Florestal, Santa Maria, RS, v. 16, n. 4, p. 437-443, 2006.

IWAKIRI, S.; MATOS, J. L. M.; PINTO, J. A.; VIANA, L. C.; SOUZA, M. M.; TRIANOSKI, R.; ALMEIDA, V. C. Produção de painéis laminados unidirecionais-LVL com lâminas de Schizolobium amazonicum, Eucalyptus saligna e Pinus taeda. Cerne, Lavras, v. 16, n. 4, p. 557-563, 2010. DOI: 10.1590/S0104-77602010000400015

IWAKIRI, S.; MATOS, J. L. M.; PRATA, J. G.; TORQUATO, L. P.; BRONOSKI, M.; NISHIDATE, M. M. Produção de painéis laminados unidirecional: LVL com madeiras de Eucalyptus grandis Hill ex Maiden e Eucalyptus dunnii Maiden. Floresta e Ambiente, Seropédica, v. 15, n. 2, p. 1-7, 2008.

IWAKIRI, S. Painéis de madeira reconstituída. Curitiba: FUPEF, 2005. 247 p.

LIMA, N. N.; MENDES, L. M.; SÁ, V. A.; BUFALINO, L. Propriedades físico-mecânicas de painéis LVL produzidos com três espécies amazônicas. Cerne, Lavras, v. 19, n. 3, p. 407-413, 2013. DOI: $10.1590 / \mathrm{S} 0104-77602013000300007$ 
MELO, R. R. Avaliação de variáveis tecnológicas na produção de painéis LVL confeccionados com paricá (Schizolobium amazonicum Huber ex. Ducke). 2012. 166 f. Tese (Doutorado em Ciências Florestais) - Universidade Federal de Brasília, Brasília, DF.

MÜlleR, M. T.; HASELEIN, C. R.; MELO, R. R. M.; STANGERLIN, D. M. Influência de diferentes combinações de lâminas de Eucalyptus saligna E Pinus taeda em painéis LVL. Ciência Florestal, Santa Maria, RS, v. 25, n. 1, p. 153-164, 2015. DOI: $10.5902 / 1980509817473$

PALMA, H. A. L.; BALLARIN, A. W. Propriedades físicas e mecânicas de painéis LVL de Eucalyptus grandis. Ciência Florestal, Santa Maria, RS, v. 21, n. 3, p. 559-566, 2011.

PEASE, D. A. Panels: products, applications and production trends. New York: M. Freeman, 1994. 254 p.
PEDROSA, A. L.; IWAKIRI, S.; MATOS, J. L. M. Produção de vigas estruturais em perfil "I" com painéis de madeira reconstituída de Pinus taeda L. E Eucalyptus dunnii Maiden. Floresta, Curitiba, v. 35, n. 3, p. 443-449, 2005. DOI: 10.5380/rf.v35i3.5189

PIO, N. S.; KEINERT JÚNIOR, S.; IWAKIRI, S.; CUNHA, U. S.; ROCHA, M. P.; LUCAS FILHO, F. C. Análise da resistência e elasticidade em flexão estática em painéis LVL de Eucalyptus grandis produzidos com lâminas pré-classificadas. Floresta, Curitiba, v. 42, n. 1 , p. $11-20,2012$. DOI: 10.5380/rf.v42i1.26288

SUCHSLAND, O. The swelling and shrinking of wood. Madison: Forest Products Society, 2004, 189 p.

TSOUMIS, G. Science and technology of wood: structure, properties and utilization. New York: Van Nostrand Reinhold, 1991. 494 p.

WALKER, J. C. F. Primary wood processing: principles and practice. London: Chapman \& Hall, 1993. 327 p. 
